\title{
Multi-lepton events and doubly-charged Higgs at HERA
}

\author{
Emmanuel Sauvan* \\ Centre de Physique des Particules de Marseille \\ 163 Avenue de Luminy F-13288 Marseille cedex 9, France \\ E-mail: sauvan@cppm.in2p3.fr
}

\section{On behalf of the $\mathrm{H} 1$ Collaboration}

Homepage: $w w w-h 1 \cdot d e s y \cdot d e$

\begin{abstract}
The production of multi-lepton events at high transverse momentum is measured in ep scattering at HERA. Within the Standard Model it proceeds mainly via photon-photon interactions. Former published analyses are extended, combining new HERA II data taken in 2003-2005 with the previous HERA I data samples. All event topologies with high $P_{T}$ electrons and muons are investigated. Yields of di-lepton and tri-lepton events are measured and a general good agreement is found with the Standard Model prediction. Events are observed with leptons of high transverse momenta in a domain where the Standard Model prediction is low.

Similar final states could also result from the single production of a double-charged Higgs boson $\left(H^{ \pm \pm}\right)$decaying into a high mass pair of same charge leptons. This possibility has been investigated and $H^{ \pm \pm}$decays involving electron, muon and taus are considered. No evidence for doubly-charged Higgs production is observed and mass-dependent upper limits are derived on the Yukawa couplings $h_{i j}$ of the Higgs boson to leptons of flavor $i$ and $j$.
\end{abstract}

International Europhysics Conference on High Energy Physics

July 21st - 27th 2005

Lisboa, Portugal

\footnotetext{
* Speaker.
} 
H1 Preliminary Multi-lepton analysis $\left(209 \mathrm{pb}^{-1}\right)$
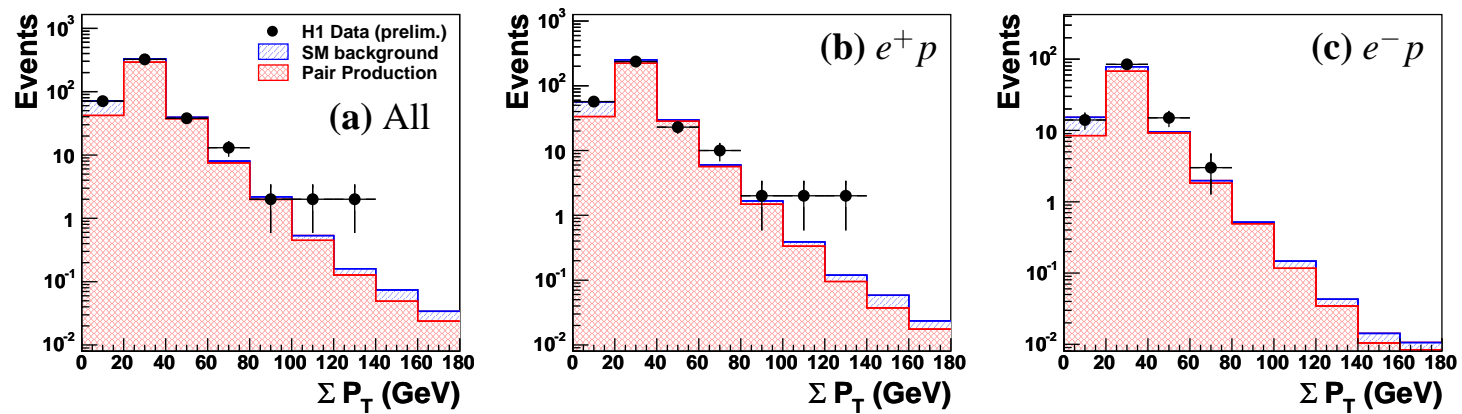

Figure 1: Distributions of the scalar sum of the transverse momenta of leptons compared to expectations for all data (a) and separatly for events recorded in $e^{+} p$ (b) and $e^{-} p$ (c) collisions.

\section{Measurement of multi-lepton events}

Within the Standard Model (SM) the production of multi-lepton events in $e p$ collisions mainly proceeds via photon-photon interactions [1]. Precise cross-section measurements of both electron (e) and muon $(\mu)$ pair production at high transverse momentum $\left(P_{T}\right)$ have already been performed by the $\mathrm{H} 1$ collaboration using the HERA I data $[2,3]$. At large di-electron masses, an excess of events was observed in both the di-electron and tri-electron samples [2]. The present analysis extends our previous measurements to the $\mathrm{e} \mu$ and $\mathrm{e} \mu \mu$ topologies and uses a higher integrated luminosity, combining new HERA II data taken in $e^{+} p$ collisions $\left(\mathscr{L}=45 \mathrm{pb}^{-1}\right)$ and in $e^{-} p$ collisions $\left(\mathscr{L}=40 \mathrm{pb}^{-1}\right)$ during the years 2003-2005 with the previous HERA I data sample from 1994-2000 $\left(\mathscr{L}=118 \mathrm{pb}^{-1}\right)$.

The multi-lepton selection requires that there be at least two central $\left(20^{\circ}<\theta<150^{\circ}\right)$ lepton (electron or muon) candidates of which one must have $P_{T}^{l}>10 \mathrm{GeV}$ and the other $P_{T}^{l}>5 \mathrm{GeV}$. Additional electron candidates are identified in the detector with an energy above $5 \mathrm{GeV}$ in the range $5^{\circ}<\theta<175^{\circ}$. Additional muons with $P_{T}>2 \mathrm{GeV}$ in the range $20^{\circ}<\theta<160^{\circ}$ are also looked for. The selected events are classified in a two lepton sample if only two central leptons are identified, and in a three lepton sample if exactly one additional lepton candidate is identified. According to the flavour of identified leptons, those samples are further classified into ee, $\mu \mu$, $\mathrm{e} \mu$, eee and $\mathrm{e} \mu \mu$. The observed event yields in all channels are in good agreement with the SM expectations which are dominated by pair production, as summarised in Tab. 1. The distribution of the scalar sum of $P_{T}\left(\sum P_{T}\right)$ of all identified leptons for the combination of di- and three-lepton samples is shown in Fig. 1a. For $\sum P_{T}>100 \mathrm{GeV} 4$ events are observed while $0.81 \pm 0.14$ are expected. These four data events correspond to the three high mass ee events observed in HERA I data [2] and one new e $\mu \mu$ event observed in the HERA II data. The separation of the data sample between events taken in collisions with a positron or electron beam is also presented in Fig. $1 \mathrm{~b}$ and Fig. 1c, respectively. All events with $\sum P_{T}>100 \mathrm{GeV}$ have been recorded in $e^{+} p$ collisions and none is shown in $e^{-} p$ data.

\section{Search for doubly-charged Higgs production}

Doubly-charged Higgs bosons $\left(H^{ \pm \pm}\right)$appear in various extensions of the SM in which the 
H1 Preliminary $209 \mathrm{pb}^{-1}(1994-2005)$

\begin{tabular}{|c|c|c|c|c|}
\hline Selection & Data & SM & Pair Production (GRAPE) & NC-DIS + Compton \\
\hline $\mathrm{ee}$ & 190 & $196 \pm 29$ & $163 \pm 17$ & $33 \pm 20$ \\
$\mu \mu$ & 82 & $85 \pm 16$ & $85 \pm 16$ & - \\
$\mathrm{e} \mu$ & 106 & $99 \pm 13$ & $61 \pm 5$ & $38 \pm 10$ \\
\hline $\mathrm{e}$ & 37 & $39 \pm 4$ & $39 \pm 4$ & $0.1 \pm 0.1$ \\
$\mathrm{e} \mu \mu$ & 50 & $51 \pm 8$ & $51 \pm 8$ & - \\
\hline
\end{tabular}

Table 1: Observed and predicted event yields for the ee, $\mu \mu$, e $\mu$, eee and $\mathrm{e} \mu \mu$ event classes. The analysed data sample corresponds to an integrated luminosity of $209 \mathrm{pb}^{-1}$. The errors on the prediction include model uncertainties and experimental systematic errors added in quadrature.

usual Higgs sector is extended by one or more triplet(s) with non-zero hypercharge $[4,5,6]$. The Higgs triplet(s) may couple to matter fields via Yukawa couplings. Whereas all charged fermions acquire their masses via their couplings to the SM Higgs doublet, the Higgs triplet(s) does not take part in the generation of fermion masses. Hence the Yukawa couplings of a $H^{ \pm \pm}$to light fermions are not constrained to be small. A non-vanishing coupling of a doubly-charged Higgs to an electron would allow its single production in $e p$ collisions at HERA. This possibility is considered and the doubly-charged Higgs decays into a high mass pair of same charge leptons are investigated.

The signal is searched for in $e e, \mu \mu, e \mu, e \tau$ and $\tau \tau$ final states. The analysis of $e e, \mu \mu$ and $e \mu$ channels is based on published analyses [2,3] and makes use of the data collected between 1994 and 2000 which amounts to a luminosity of $118 \mathrm{pb}^{-1}$. The analysis of final states involving a $\tau$ lepton is based on $88 \mathrm{pb}^{-1}$ of $e^{+} p$ data recorded during the same period. After the final Higgs selection criteria no significant excess over the SM expectation is observed in the data. Amongst the six di-electron events at $M_{e e}>100 \mathrm{GeV}$ observed in [2] only one satisfies all the $H^{ \pm \pm}$selection criteria.

The resulting constraints assuming that the doubly-charged Higgs boson only decays to electrons are shown in Fig. 2a. A lower limit on $M_{H}$ of $139 \mathrm{GeV}$ is obtained for $h_{e e}=0.3$. The result is compared to the bounds obtained from searches for $H^{ \pm \pm}$pair production at LEP [7], by the CDF experiment [8], and to both the indirect and direct limits obtained by the OPAL experiment [9]. From the $e^{ \pm} p \rightarrow \mu^{\mp} H^{ \pm \pm} X \rightarrow \mu^{\mp} e^{ \pm} \mu^{ \pm} X$ and $e^{ \pm} p \rightarrow \tau^{\mp} H^{ \pm \pm} X \rightarrow \tau^{\mp} e^{ \pm} \tau^{ \pm} X$ analyses upper limits on $h_{e \mu}$ and $h_{e \tau}$ are derived. Assuming that the doubly-charged Higgs boson decays only into electron-muon (electron-tau), a lower limit on $M_{H}$ of $140 \mathrm{GeV}(112 \mathrm{GeV})$ is obtained for $h_{e \mu}=0.3$ $\left(h_{e \tau}=0.3\right)$. The results are shown in Fig. $2 \mathrm{~b}$ and Fig. $2 \mathrm{c}$, and are compared to limits from direct searches for $H^{ \pm \pm}$pair production from CDF [8] and the LEP experiments [7]. The H1 limits extend the excluded region in the $e \mu$ and $e \tau$ channels to masses that are beyond those reached in previous searches.

\section{Conclusions}

The production of multi-leptons (electrons and muons) at high transverse momenta in $e p$ scattering has been studied. The measurement extends previous analyses [2,3] by including the HERA II data recorded in $e^{+} p$ and $e^{-} p$ collisions and corresponding to integrated luminosities 

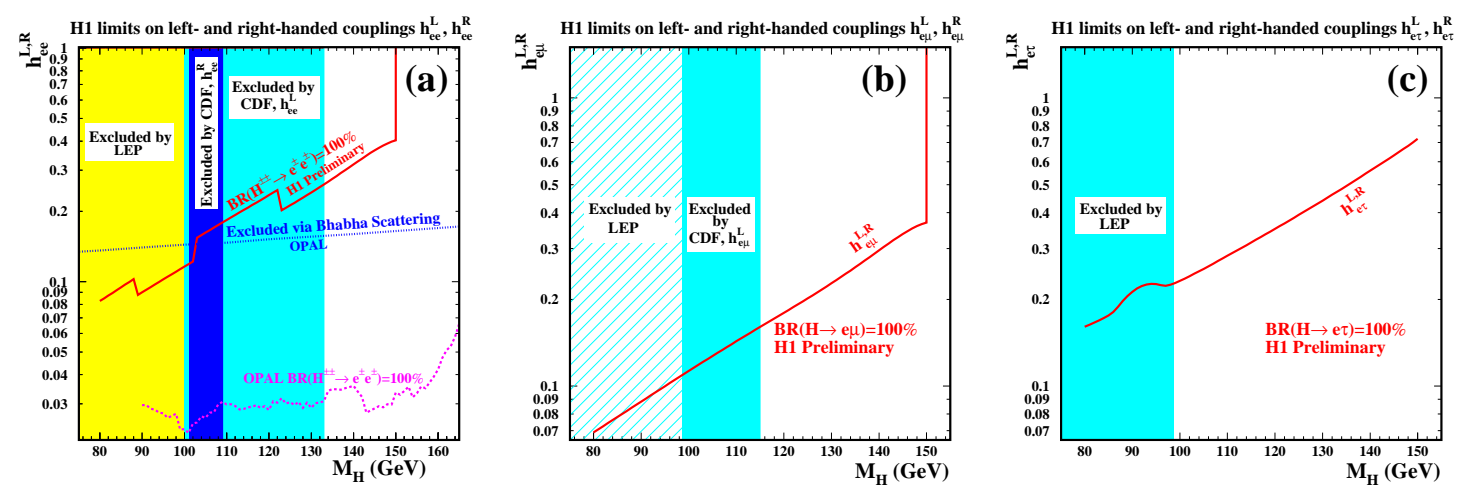

Figure 2: Upper limits at $95 \%$ confidence level on the coupling $h_{e e}$ as a function of the doubly-charged Higgs mass assuming that the $H^{ \pm \pm}$only couples to electrons (a). Regions above the curves are excluded. In (b) and (c) upper limits on the couplings $h_{e \mu}$ and $h_{e \tau}$ are shown, assuming that the $H^{ \pm \pm}$only couples to $e \mu$ and $e \tau$, respectively.

luminosity of $45 \mathrm{pb}^{-1}$ and $40 \mathrm{pb}^{-1}$, respectively. The event yields in the di-lepton (ee, $\mu \mu$ and $\mathrm{e} \mu$ ) and tri-lepton (eee and e $\mu \mu$ ) classes are in good agreement with the SM predictions. The distribution of the scalar sum of transverse momenta of the leptons is studied for the combination of all diand tri-lepton events. For $\sum P_{T}>100 \mathrm{GeV} 4$ events are observed and $0.81 \pm 0.14$ are expected. A search for the single production of doubly-charged Higgs bosons is also presented which includes the analysis of the $e e, \mu \mu, e \mu, \tau \tau$ and $e \tau$ final states. New limits on the $H^{ \pm \pm}$mass and its Yukawa coupling to electrons, muons and taus are obtained. Assuming that the doubly-charged Higgs only decays to electrons, we set a lower limit on $M_{H}$ of about $139 \mathrm{GeV}$ for a coupling value $h_{e e}=0.3$. Assuming that the doubly-charged Higgs only decays to electron-muon (electron-tau), we set a lower limit on $M_{H}$ of about $140 \mathrm{GeV}(112 \mathrm{GeV})$ for a coupling value $h_{e \mu}=0.3\left(h_{e \tau}=0.3\right)$.

\section{References}

[1] J.A.M. Vermaseren, Nucl. Phys. B 229, (1983) 347;

N. Artega-Romero, C. Carimalo and P. Kessler, Zeit.f.Phys C 52, (1991) 289.

[2] A. Aktas et al. [H1 Collaboration], Eur. Phys. J. C 31 (2003) 17 [hep-ex/0307015].

[3] A. Aktas et al. [H1 Collaboration], Phys. Lett. B 583 (2004) 28 [hep-ex/0311015].

[4] G. B. Gelmini and M. Roncadelli, Phys. Lett. B 99 (1981) 411.

[5] J. C. Pati and A. Salam, Phys. Rev. D 10 (1974) 275;

R. E. Marshak and R. N. Mohapatra, Phys. Lett. B 91 (1980) 222.

[6] R. N. Mohapatra and G. Senjanovic, Phys. Rev. Lett. 44 (1980) 912.

[7] J. Abdallah et al. [DELPHI Collaboration], Phys. Lett. B 552 (2003) 127 [hep-ex/0303026];

P. Achard et al. [L3 Collaboration], Phys. Lett. B 576 (2003) 18 [hep-ex/0309076];

G. Abbiendi et al. [OPAL Collaboration], Phys. Lett. B 526 (2002) 221 [hep-ex/0111059].

[8] D. Acosta et al. [CDF Collaboration], Phys. Rev. Lett. 93 (2004) 221802 [hep-ex/0406073].

[9] G. Abbiendi et al. [OPAL Collaboration], Phys. Lett. B 577 (2003) 93 [hep-ex/0308052]. 